\title{
Preliminary clinical and radiographic outcomes of oblique lateral interbody fusion combined with percutaneous transforaminal endoscopic discectomy for degenerative lumbar disease accompanied by prolapsed disc herniation
}

\section{Chao Lou}

Lishui Central Hospital and Fifth Affiliated Hospital of Wenzhou Medical College

Shijie Liu

Lishui Central Hospital and Fifth Affiliated Hospital of Wenzhou Medical College

\section{Weiyang Yu}

Lishui Central Hospital and Fifth Affiliated Hospital of Wenzhou Medical College

Feijun Liu

Lishui Central Hospital and Fifth Affiliated Hospital of Wenzhou Medical College

Zhenzhong Chen

Lishui Central Hospital and Fifth Affiliated Hospital of Wenzhou Medical College Jiawei Gao

Lishui Central Hospital and Fifth Affiliated Hospital of Wenzhou Medical College Bin Pan

Lishui Central Hospital and Fifth Affiliated Hospital of Wenzhou Medical College Dengwei He ( $\nabla$ hedw_spine@163.com )

Wenzhou Medical University Eye Hospital

\section{Research article}

Keywords: lumbar disc disease, minimally invasive, oblique lumbar interbody fusion, percutaneous transforaminal endoscopic discectomy

Posted Date: February 18th, 2020

DOI: https://doi.org/10.21203/rs.2.23816/v1

License: (c) (i) This work is licensed under a Creative Commons Attribution 4.0 International License. Read Full License 


\section{Abstract}

Objective: To investigate the preliminary clinical and radiographic outcomes of oblique lateral interbody fusion (OLIF) combined with percutaneous transforaminal endoscopic discectomy (PTED) for the degenerative lumbar disease accompanied by prolapsed disc herniation.

Methods: From March 2016 to December 2018, 15 patients with degenerative lumbar spondylolisthesis, lumbar spine instability or lumbar spinal stenosis accompanied by prolapsed disc herniation were underwent OLIF combined with PTED in our spine surgery center, including 6 males and 9 females, the mean age was $61.4 \pm 7.1$ years. Clinical results, radiological parameters, and related complications were collected and analyzed.

Results: All patients firstly received PTED with local anesthesia and then underwent OLIF with general anesthesia. All patients were followed up for an average of $14.6 \pm 3.7$ months. Mean preoperative visual analog scale scores and Oswestry Disability Index scores were significantly improved postoperatively $(P<0.05)$. The radiographic results include the intervertebral disc height, vertical diameter of intervertebral foramen, spinal canal anteroposterior diameter, segment angle and lumbar lordosis angle were significantly improved postoperatively, respectively $(P<0.05)$. None major implant-related and other severe complications were happened.

Conclusions: OLIF combined with PTED can successfully enables direct neural decompression without posterior decompressive procedures and might be an alternative minimally invasive surgical option for properly selected patients.

\section{Introduction}

Lumbar interbody fusion has been the most general and effective surgical method for degenerative lumbar disease..$^{1-3}$ Due to conventional open procedures cause great injury to posterior musculoskeletal structure, minimally invasive techniques for lumbar fusion have been developed with good clinical outcomes and fewer complications compared with open procedures. ${ }^{4-7}$ More recently, oblique lateral interbody fusion (OLIF), with anteropsoas exposure, have been applied for the treatment of lumbar degenerative disease, for its priority of less psoas and lumbar plexus injury, trauma, blood loss and enhanced postoperative recovery. ${ }^{8-11}$

Spinal canal is not accessible in the process of OLIF, and direct decompression can not be achieved, which may restrict the application of OLIF in the patients accompanied by prolapsed lumbar disc herniation or severe lumbar spinal stenosis. ${ }^{12}$ However, the indications for OLIF might be extended when it combined with the assistance of spinal endoscopic discectomy, that direct decompression for intervertebral foramen or spinal canal would be realized. ${ }^{12,13}$

So far, spinal endoscopic discectomy-assisted OLIF has been rarely reported and there are several limitations in previous studies. ${ }^{12-14}$ In present study, we retrospectively analyzed 15 patients who had 
degenerative lumbar spondylolisthesis, lumbar spine instability or lumbar spinal stenosis accompanied by prolapsed disc herniation, and receieved treatment of OLIF combined with percutaneous transforaminal endoscopic discectomy (PTED). The purpose of our study was to assess the clinical and radiological outcomes of patients treated with OLIF combined with PTED.

\section{Materials And Methods}

\section{Study Population}

From March 2016 to December 2018, 176 patients were underwent OLIF procedure at our institution and with a minimum of 12 months of follow-up. Among these patients, 15 patients were underwent OLIF combined with PTED and enrolled for this study. Indications for OLIF combined with PTED were degenerative lumbar spondylolisthesis, lumbar spine instability or lumbar spinal stenosis with a concomitant prolapsed herniation disc. Patients with resting radicular pain and low back pain or neurogenic intermittent claudication were considered to have a concomitant prolapsed herniation disc and were included for OLIF combined with PTED. Before surgical intervention was advised, conservative treatment for over 3 months failed in these patients. This study was approved by the institutional review board of our institution. Additionally, informed written consents were obtained from all patients.

\section{Operative procedures}

First, the PTED procedure was performed in a lateral decubitus position under local anesthetics and intravenous sedation. Patients could freely communicate with the surgeon during the entire procedure. By target puncture, operating channel was placed and intervertebral foramen was reformed. The end point of the procedure is that free mobilization of neural tissue is achieved, thereafter the endoscope is withdrawn and a sterile dressing is applied over a one-point subcutaneous suture (Figs. 1d-f).

After PTED, the patient was received induction of general anesthesia and placed in a right-sided lateral decubitus position. All patients underwent standard OLIF procedure (Figs. $1 \mathrm{~g}$-i). ${ }^{15,16} \mathrm{In}$ addition, posterior lumbar stabilization was performed with percutaneous pedicle screw fixation after OLIF for the patients with osteoporosis ( $T$ value of bone mineral density is less than or equal to -2.5 ) or instability according to preoperative flexion-extension radiograph.

\section{The assessment of radiological and clinical outcomes}

General information were collected, including operation duration and blood loss of OLIF and PTED, intraoperative and postoperative complications, duration of postoperative hospital stay. Visual analogue scale (VAS) was used to assess low back and leg pain. Oswestry disability index (ODI) ${ }^{17}$ was used to evaluate the disability status preoperatively and at the last follow-up.

The radiological parameters that we analyzed were diagnosis, operative level(s), intervertebral disc height, intervertebral foramen height, spinal canal diameter, segmental lordosis angle and whole lumbar lordotic 
angle. Intervertebral disc height and spinal canal diameter were measured in midsaggital T2-weighted MR imaging. Then intervertebral disc height, segmental lordosis angle and whole lumbar lordotic angle were measured in X-ray film. Cage location and fusion in intervertebral space were observed by postoperative CT scanning. All imaging measurements were obtained with a picture archiving and communication systems (PACS, RADinfo, Hangzhou, China). All the data were assessed by 2 senior spine surgeons who were blinded to the situation

\section{Statistical analysis}

The statistical analysis was performed with SPSS version 19.0 (SPSS, Chicago, Illinois, USA). Quantitative results were expressed in terms of their mean and standard deviation. Statistical analysis was performed using the Wilcoxon rank sum test. A P value less than 0.05 was considered statistically significant.

\section{Results}

The mean age of the 15 patients ( 6 males and 9 females) was $61.4 \pm 7.1$ years (range, $52-75$ years). Two cases were at L3-4 level, while other 13 cases were at L4-5 level (Table 1). The mean operation time of PTED was $55.7 \pm 13.9 \mathrm{~min}$ ( range, $35-85 \mathrm{~min}$ ), and the mean blood loss was $11.7 \pm 5.7 \mathrm{ml}$ (range, 5$25 \mathrm{ml}$ ), while the mean operation time of OLIF was 60.3 $\pm 22.3 \mathrm{~min}$ (range, 40-120 min), with the mean blood loss was $67.3 \pm 22.9 \mathrm{ml}$ (range, $30-120 \mathrm{ml}$ ). The mean duration of postoperative hospital stay was $7.6 \pm 1.1$ days (range, $6-10$ days). The mean follow up was $14.6 \pm 3.7$ months (range, $12-20$ months) (Table 1).

All 15 patients firstly received PTED surgery with local anesthesia, and following OLIF surgery with general anesthesia. Among them, there were 5 cases of central prolapsed disc herniation (33.3\%) and 10 cases of foraminal prolapsed disc herniation (66.7\%), and herniated disc were removed in PTED surgery successfully. Another, 6 cases were underwent percutaneous pedicle screw fixation (40\%). The mean preoperative VAS score of low back pain and leg pain significantly improved from $6.5 \pm 1.3$ to $1.3 \pm 0.7$ and $8.3 \pm 0.8$ to $1.1 \pm 0.4$ at the final follow-up, respectively $(P<0.05)$. In addition, the mean preoperative ODI value also significantly improved from $86.7 \pm 15.7 \%$ to $14.5 \pm 5.2 \%$ at the final follow-up $(p<0.05)$ (Table 2).

The mean diameter of central canal increased signifcantly from $6.1 \pm 1.3 \mathrm{~mm}$ to $10.4 \pm 2.4 \mathrm{~mm}(P<0.05)$. The height of intervertebral disc increased signifcantly from $7.0 \pm 1.2 \mathrm{~mm}$ to $11.5 \pm 1.6 \mathrm{~mm}(P<0.05)$ as well as that of intervertebral foramen, increased signifcantly from $15.1 \pm 2.1 \mathrm{~mm}$ to $19.2 \pm 2.5 \mathrm{~mm}(\mathrm{P}<$ 0.05). Besides, a signifcant postoperative increase was observed also in mean segmental lordosis angle (from $10.3 \pm 3.4^{\circ}$ to $\left.19.4 \pm 7.8^{\circ}\right)(P<0.05)$ and whole lumbar lordotic angle (from $16.4 \pm 6.5^{\circ}$ to $27.5 \pm 8.3^{\circ}$ ) $(P<0.05)$ (Table 3).

No operation-related severe complication occurred in all cases. Transient anterolateral thign numbness happened in 1 case (6.7\%), while weakness in hip flexion was observed in 1 case (6.7\%) and relieved at 1 
month follow-up visit. Among 9 case with stand-alone OLIF, cage subsidence for over $2 \mathrm{~mm}$ were observed in 1 case, and the main complaint was intolerable low back pain, and finally relieved after receiving percutaneous pedicle screw fixation.

\section{Discussion}

OLIF is a minimally invasive lumbar interbody fusion and has been proved an effective treament for degenerative lumbar disease. ${ }^{8-11}$ Compared with transforaminal lumbar interbody fusion (TLIF) or posterior lumbar interbody fusion (PLIF), OLIF can maximally preserve posterior structures, including ligamentous structures, muscle and facet joints, however, OLIF does not access to spinal canal and the resulting indirect decompression is a drawback and restricts the application of OLIF, expecially for lumbar degenerative disease accompanied by prolapsed disc herniation or severe lumbar spinal stenosis, which are contraindications of OLIF. ${ }^{12,13}$ For the above situations, surgeons always choose traditional TLIF or PLIF for direct decompression. However, the risk of substantial bleeding, direct neural injury, epidural adhesion, and posterior ligamentous injury are disadvantages of TLIF and PLIF. We recently attempted OLIF combined with PTED for treating lumbar degenerative disease accompanied by prolapsed disc herniation. PTED is an effective minimally invasive surgery and has been well accepted by surgeons and patients in addressing lumbar disc herniation. PTED has multiple advantages, including local anesthesia, less tissue damage, less risk of nerve injury, shorter operative time and faster recovery. ${ }^{18,19}$ Our results indicated that OLIF combined with PTED could significantly relieve the low back and leg pain, improve the disabilities of patients with lumbar degenerative disease accompanied by prolapsed disc herniation. Moreover, there were only 3 patients experiencing complications, but they relieved at follow-up visit, which manifested OLIF combined with PTED was safety for properly selected patients.

Some researchers have also evaluated the clinical and radiographic effectiveness of spinal endoscopic discectomy-assisted OLIF. ${ }^{12,13}$ Heo et al ${ }^{12}$ had reported OLIF in assistance of endoscopy in same working channel to treat 14 cases of degenerative lumbar disease with lumbar disc herniation. Compared with their technique, though we needed to change patient position intraoperation and an extra incision was made for PTED, our technique also had multiple advantages: Firstly, the indications for spinal endoscopic discectomy-assisted OLIF in their study was only for degenerative lumbar disease with right foraminal or central disc herniation, as patients were set in right lateral postion and working channel is set by left approach. Our technique is able to decompress spinal canal in all directions, decompressive region is enlarged and indications of endoscopic-assisted OLIF are expanded. Secondly, general anesthesia was kept in their whole process and the mean general anesthesia (refer to operation time) was $155.8 \pm 45.1 \mathrm{~min}$. While in our technique, the PTED procedure was performed under local anesthesia, and general anesthesia was only carried in OLIF procedure and the mean general anesthesia (refer to operation time) of OLIF was $60.3 \pm 22.3 \mathrm{~min}$. Signifcant reduction of general anesthesia time would reduce the perioperative risk especially for the elderly and accelerate rehabilitation postoperation, which is very valuable for the patients. Thirdly, as the patient remained awake in PTED, and favorable feedback of patient for nerve stimulation helped reducing the risk of nerve injury. Lastly, endoscopy had limited view 
and needed assistance of special surgical instruments with endoscopic discectomy was conducted in channel of OLIF, however, PTED was conducted by intervertebral foramen approach, which was more feasible and familiar to most surgeons. Heo et al ${ }^{12}$ had reported another advantage in their study was that being able to extend the fusion bed through endoscopic discectomy and explore the endplate through endoscopic visualization, however, the differences in the fusion rate and clinical outcomes between traditional OLIF with spinal endoscopic discectomy-assisted OLIF were unclear and have not been studied. Therefore, further studies are needed to be carried out to investigate these issues.

Though ALIF can also achieved indirect and direct decompression by removing herniated disc under direct version, it has a long learning curve and cause the possibility of significant complications like iliac vessel and peritoneal injury. ${ }^{20,21}$ Compared with those of TLIF or PLIF procedures, the present study indicated that OLIF combined with PTED could greatly reduced operation time and blood loss, which was in correspondence with conclusions of Heo et al. ${ }^{12}$ In our experience, the mean duration of PTED was performed only $55.7 \pm 13.9 \mathrm{~min}$, and mean blood loss was only $11.7 \pm 5.7 \mathrm{ml}$. No complications associated with PTED were encountered. The risk of lumbar plexus injury is unignorable with lateral lumbar interbody fusion (LLIF). Bergey et al reported that $30 \%$ of the patients exhibited paresthesia, thigh pain, and lumbar plexus injury afer surgery using the direct lateral approach. ${ }^{22}$ The approach of OLIF was performed between the anterior vessels and the psoas muscles, and the occurrence of lumbar plexus injuriy was reduced. In the present study, we found only minor injury or irritation of the lumbar plexus in 2 patients, and these complications were relieved at 1 month follow-up visit.

OLIF combined with PTED have particular advantages, and expand indications of OLIF technique. As PTED working in a new channel and decompressing ventral dura in all directions, it was capable of treating degenerative disease like vertebral posterior osteophytes, and migrated disc herniation and intervertebral disc calcification, while these special situations were limited by Heo's technique. ${ }^{12}$ However, special attention should also be paid in our technique. On one hand, to perform OLIF combined with PTED, previous experience with PTED is required. In our patients, additional PTED was performed by an expert endoscopic spine surgeon. On the other hand, OLIF combined with PTED has limitation in decompressing dorsal dura, it may not be eligible for facet joint hypertrophy and ligamentum flavum calcification or ossification.

Nevertheless, several potential study limitations also need to be acknowledged. Firstly, the study was retrospective by design and had a small sample size, however, the incidence of such diseases is relative low. Besides, our technique still required randomized controlled trial with other interbody fusion future. More importantly, for the patients underwent OLIF along with PTED and percutaneous pedicle screw fixation, though these techniques are all minimally invasive, they together may increased operation time. Further randomized studies with a larger sample size and a longer follow-up period are needed to demonstrate this issue.

\section{Conclusions}


OLIF combined with PTED can successfully enables direct neural decompression without posterior decompressive procedures and provide a satisfactory outcome. It can overcome the limitations of OLIF and might be an alternative minimally invasive surgical option for properly selected patients.

\section{Abbreviations}

ODI - Oswestry disability index

OLIF- Oblique lateral interbody fusion

PLIF- Posterior lumbar interbody fusion

PTED-Percutaneous transforaminal endoscopic discectomy

TLIF-Transforaminal lumbar interbody fusion

VAS - Visual analog scale

\section{Declarations}

\section{Acknowledgements}

No

\section{Ethical review committee statement}

Ethical approval was obtained from the Medical Ethics Committee of our hospital. Additionally, all patients gave written informed consent for their information to be stored in the hospital's database and used for research.

\section{Authors' contributions}

All authors were involved in conception and design. CL, SJL, WYY, and DWH contributed to study design. FJL, ZZC, and JWG contributed to study conduct. WYY, FJL, and BP contributed to data collection and analysis. CL, WYY, and DWH contributed to data interpretation. CL, SJL, and DWH contributed to drafting of the manuscript. All authors take responsibility for the integrity of the data analysis. All authors read and approved the final manuscript.

\section{Funding}

This study was partially supported by The Zhejiang Province Natural Science Foundation of China (LY17C100002), The Zhejiang Experimental Animal and Technology Program Foundation of China (2018C37099), The Zhejiang Province Scientifc Project of Health and Medicine of China (2018KY936).

\section{Availability of data and materials}

The datasets used and analyzed during the current study are available from the corresponding author on reasonable request. 


\section{Ethics approval and consent to participate}

The study was reviewed and approved by the institutional review board and the ethics committee of our institution. Patients or their family members agreed to our study, and signed the informed consents.

\section{Consent for publication}

Not applicable

\section{Competing interests}

The authors declare that they have no competing interests

\section{References}

1. Mummaneni PV, Dhall SS, Eck JC, Groff MW, Ghogawala Z, Watters WC 3rd, et al. Guideline update for the performance of fusion procedures for degenerative disease of the lumbar spine. Part 11: interbody techniques for lumbar fusion. J Neurosurg Spine. 2014;21(1): 67-74.

2. Resnick DK, Watters WC 3rd, Sharan A, Mummaneni PV, Dailey AT, Wang JC, et al. Guideline update for the performance of fusion procedures for degenerative diseaseof the lumbar spine. Part 9: lumbar fusion for stenosis with spondylolisthesis. J Neurosurg Spine. 2014; 21(1): 54-61.

3. de Kunder SL, van Kuijk SMJ, Rijkers K, Caelers IJMH, van Hemert WLW, de Bie RA, et al. Transforaminal lumbar interbody fusion (TLIF) versus posterior lumbar interbody fusion (PLIF) in lumbar spondylolisthesis: a systematic review and meta-analysis. Spine J.2017; 17(11): 1712-21.

4. Foley KT, Holly LT, Schwender JD. Minimally invasive lumbar fusion. Spine (Phila Pa 1976). 2003; 28 (15 Suppl):S26-S35.

5. Park Y, Ha JW. Comparison of one-level posterior lumbar interbody fusion performed with a minimally invasive approach or a traditional open approach. Spine (Phila Pa 1976). 2007; 32(5):53743.

6. Mobbs RJ, Phan K, Malham G, Seex K, Rao PJ. Lumbar interbody fusion: techniques, indications and comparison of interbody fusion options including PLIF, TLIF, MIQTLIF, OLIF/ATP, LLIF and ALIF. J Spine Surg. 2015; 1(1): 2-18.

7. Kotwal S, Kawaguchi S, Lebl D, Hughes A, Huang R, Sama A, et al. Minimally Invasive Lateral Lumbar Interbody Fusion: Clinical and Radiographic Outcome at a Minimum 2-year Follow-up. J Spinal Disord Tech.2015; 28(4): 119-25.

8. Ohtori S, Orita S, Yamauchi K, Eguchi Y, Ochiai N, Kishida S, et al. Mini-open anterior retroperitoneal lumbar interbody fusion: oblique lateral interbody fusion for lumbar spinal degeneration disease. Yonsei Med J. 2015;56(4):1051-9

9. Fujibayashi S, Hynes RA, Otsuki B, Kimura H, Takemoto M, Matsuda S. Effect of indirect neural decompression through oblique lateral interbody fusion for degenerative lumbar disease. Spine (Phila Pa 1976). 2015;40(3):E175-E182. 
10. Woods KR, Billys JB, Hynes RA. Technical description of oblique lateral interbody fusion at L1-L5 (OLIF25) and at L5-S1 (OLIF51) and evaluation of complication and fusion rates. Spine J. 2017; 17(4): 545-53.

11. Mehren C, Mayer HM, Zandanell C, Siepe CJ, Korge A. The Oblique anterolateral approach to the lumbar spine provides access to the lumbar spine with few early complications. Clin Orthop Relat Res. 2016; 474(9): 2020-7.

12. Heo DH, Choi WS, Park CK, Kim JS. Minimally invasive oblique lumbar interbody fusion with spinal endoscope assistance: technical note. World Neurosurg. 2016; 96: 530-6.

13. Heo DH, Kim JS. Clinical and radiological outcomes of spinal endoscopic discectomy-assisted oblique lumbar interbody fusion:preliminary results. Neurosurg Focus. 2017; 43 (2): E13.

14. Kim JS, Seong JH. Endoscope-assisted oblique lumbar interbody fusion for the treatment of cauda equina syndrome: a technical note. Eur Spine J. 2017; 26(2):397-403.

15. Zeng ZY, Xu ZW, He DW, Zhao X, Ma WH, Ni WF, et al. Complications and prevention strategies of oblique lateral interbody fusion technique. Orthop Surg. 2018; 10(2): 98-106.

16. Liu J, He Y, Huang B, Zhang X, Shan Z, Chen J, et al. Reoccurring discogenic low back pain (LBP) after discoblock treated by oblique lumbar interbody fusion (OLIF). J Orthop Surg Res. 2020, 15(1): 22.

17. Fairbank JC, Pynsent PB. The Oswestry Disability Index. Spine (Phila Pa 1976). 2000; 25(22):294052.

18. Gadjradj PS, van Tulder MW, Dirven CM, Peul WC, Harhangi BS. Clinical outcomes after percutaneous transforaminal endoscopic discectomy for lumbar disc herniation: a prospective case series. Neurosurg Focus. 2016; 40(2): E3.

19. Choi KC, Kim JS, Lee DC, Park CK. Percutaneous endoscopic lumbar discectomy: minimally invasive technique for multiple episodes of lumbar disc herniation. BMC musculoskeletal disorders. 2017; 18(1):329.

20. Rao PJ, Loganathan A, Yeung V, Mobbs RJ. Outcomes of anterior lumbar interbody fusion surgery based on indication: a prospective study. Neurosurgery. 2015;76(1):7-23.

21. Rao PJ, Ghent F, Phan K, Lee K, Reddy R, Mobbs RJ. Stand-alone anterior lumbar interbody fusion for treatment of degenerative spondylolisthesis. J Clin Neurosci. 2015;22(10):1619-24.

22. Bergey DL, Villavicencio AT, Goldstein T, Regan JJ. Endoscopic lateral transpsoas approach to the lumbar spine. Spine (Phila Pa 1976). 2004;29(15):1681-8.

\section{Tables}


Table 1. General information of 15 cases

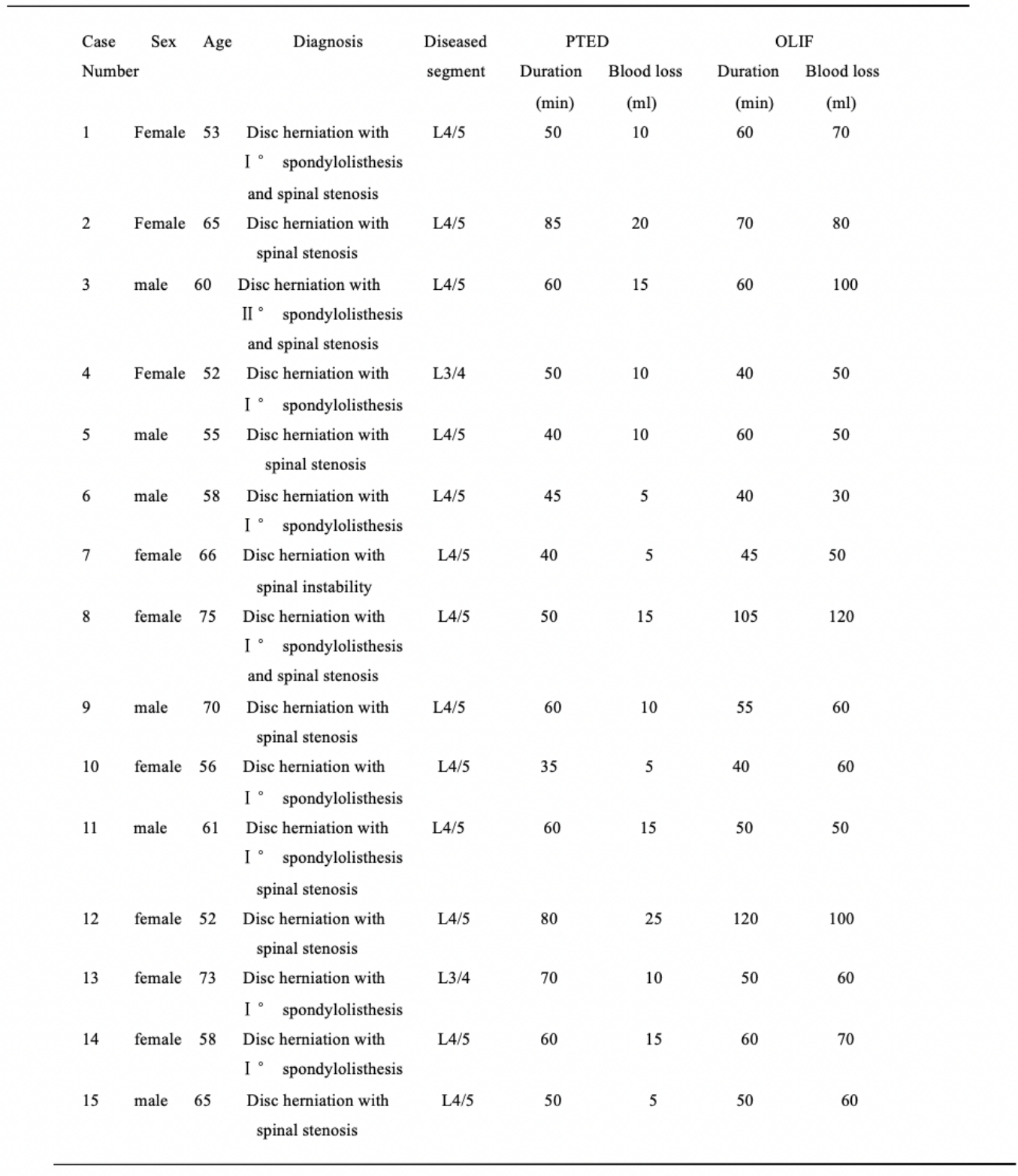


Table 2. Preoperative and postoperative clinical results evaluation of 15 cases

\begin{tabular}{lccc}
\hline & & & \\
& preoperation & last follow-up & P \\
\hline VAS of low back pain & $6.5 \pm 1.3$ & $1.3 \pm 0.7$ & $<0.001$ \\
VAS of leg pain & $8.3 \pm 0.8$ & $1.1 \pm 0.4$ & $<0.001$ \\
ODI (\%) & $86.7 \pm 15.7$ & $14.5 \pm 5.2$ & $<0.001$ \\
\hline
\end{tabular}

The data are showed as means \pm standard deviation (SD) values.

VAS - Visual analog scale ; ODI - Oswestry disability index

Table 3. Preoperative and postoperative radiograhic parameters evaluation of 15 cases

\begin{tabular}{lclc}
\hline & & & \\
& Preoperation & Postoperation & $\mathrm{P}$ \\
\hline Central canal diameter(mm) & $6.1 \pm 1.3$ & $10.4 \pm 2.4$ & $<0.001$ \\
Intervertebral disc height(mm) & $7.0 \pm 1.2$ & $11.5 \pm 1.6$ & $<0.001$ \\
Intervertebral foramen height(mm) & $15.1 \pm 2.1$ & $19.2 \pm 2.5$ & $<0.001$ \\
Diseased segment lordotic angle $\left(^{\circ}\right)$ & $10.3 \pm 3.4$ & $19.4 \pm 7.8$ & $<0.001$ \\
Whole lumbar lordotic angle $\left(^{\circ}\right)$ & $16.4 \pm 6.5$ & $27.5 \pm 8.3$ & $<0.001$ \\
\hline
\end{tabular}

The data are showed as means \pm standard deviation (SD) values.

\section{Figures}



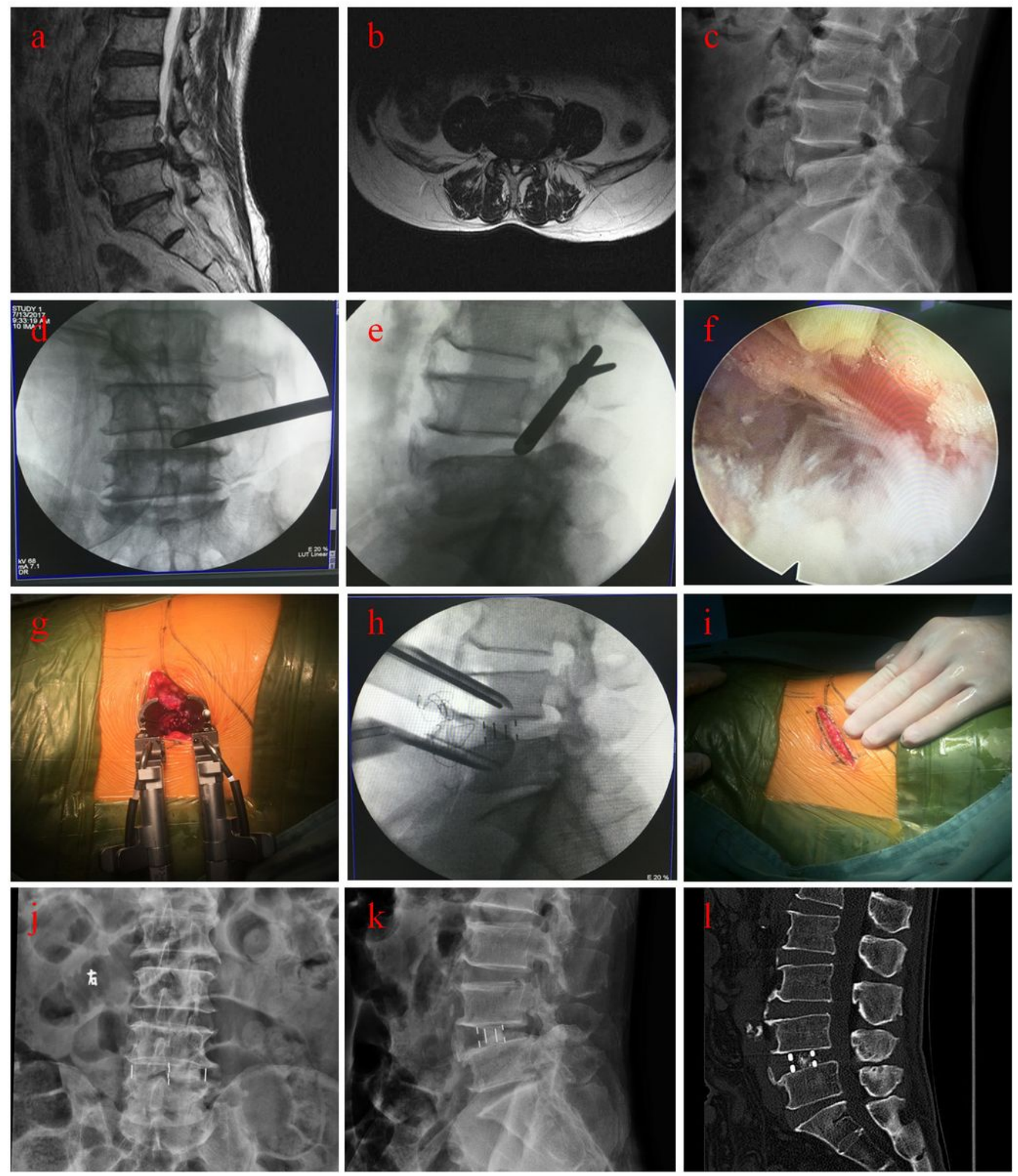

\section{Figure 1}

Male, 53 years old, complained low back pain with neurogenic intermittent claudication and radicular pain of right lower extremity, and the radicular leg pain did not relieved after resting. The patient suffered from I'lumbar degeneration spondylolisthesis, spinal stenosis with a concomitant right intervertebral foramen type prolapsed disc herniation (a-c), and then received PTED removing diseased nucleus pulposus in local anesthesia (d-f) and OLIF in general anesthesia ( $\mathrm{g}-\mathrm{i})$. After surgery, the position of cage 
was immediately presented in anteroposterior and lateral film (j,k). By 3 months postoperation, CT scanning showed stable position of cage and partial synostosis formation (I). 